Orissa Journal of Commerce

Vol. 42, Issue 1, Jan-March 2021

ISSN: 0974-8482

(C) OJC India. All Right Reserved

URL : www.ojcoca.org

DOI: https://doi.org/10.54063/ojc.2021.v42i01.05

\title{
Asset Quality Evaluation of Select Indian Banks: A Camel Model
}

\author{
Priyanka Jha $^{1^{*}}$ and Subburaj Natarajan ${ }^{2}$ \\ ${ }^{1}$ Research Scholar, Department of Management, Sarvepalli Radhakrishnan University, Bhopal, Madhya Pradesh. \\ E-mail:pari4337@gmail.com \\ ${ }^{2}$ Associate Professor, Department of Management, Sarvepalli Radhakrishnan University, Bhopal, Madhya Pradesh. \\ E-mail:subburajmba2010@gmail.com \\ ${ }^{*}$ Corresponding Author
}

To cite this paper

Jha, P., \& Natarajan, S. (2021). Asset Quality Evaluation of Select Indian Banks: A Camel Model. Orissa Journal of Commerce. 42(1), 59-77.

Keywords

CAMEL, Commercial banks, Non-performing assets, Financial performance, Asset quality.

JEL Classification E51, F65, G21, G23, G33

\begin{abstract}
Banking Sector has a direct impact on the economic growth of a country. The performance of Bank is analysed in regular basis. The 'CAMEL' model is a reliable and precise method to use as a performance assessor in the Banking Industry and to anticipate potential and relative risk. The present study is being conducted to make a comparative analysis of the financial performance of State Bank of India (SBI) and Punjab National Bank (PNB) in Public Sector and Industrial Credit and Investment Corporation of India (ICICI) and AXIS Bank (AXIS) in Private Sector during 2011-2018 with CAMEL Model (Capital Adequacy, Assets Quality, Productivity of Management, Earnings Quality, and Liquidity). The financial performance of a bank is analyzed on the basis of CAMEL rating and it ranked accordingly. The data is collected from the respective Bank's annual reports. The overall performance of the ICICI is excellent, and the Bank is ranked (1) among four Banks. AXIS is ranked (2) followed by SBI (3) and PNB (4). However PNB is poorly performed in the overall composite ranking. The Overall financial performance of Public Sector Banks is not up to the mark as compare to Private Sector Bank and to be tracked closely to ensure their viability.
\end{abstract}

\section{Introduction}

The soundness of the financial sector has a direct impact on the economic growth of a country. Banks is major contributors in it. The performance of Bank is analysed by the Reserve Bank of India, in terms of Capital Adequacy, Assets Quality, Management Efficiency, Earnings Quality, and Liquidity to regulate the level of risk and financial viability of Commercial Banks. There are several methods to analyse the performance of a Bank. In Assets Quality, the value of assets is compared in the balance sheet. Bank executives are concerned with the quality of their loans, as they provide Banks with revenue. Banks' Management Efficiency represents the ability to identify, evaluate, track, and control the risk to be handled. To see consistency in the Bank's profits, earnings ratios are determined. Liquidity refers to the capacity of Banks to fulfil their short-term commitments. Those extending short-term credit to 
the company is particular concern to them. An adequate Liquidity condition means a situation in which an entity can obtain ample liquid funds by increasing its liabilities or rapidly turning its assets into Cash. This analysis focuses on analysing the Indian Bank's financial viability and Banking adequacy. In this study, the CAMEL Model is being used. The CAMEL Model is a ratio-based method for understanding Bank output under different criteria (Getahun, 2015). CAMEL has Sub-parameters Capital Adequacy, Assets Quality, Management Capability, Earning Efficiency and Liquidity by which Bank output is calculated. The CAMEL Model demonstrates the performance of Assets, Profitability, and Liquidity. It also provides the flexibility to choose the most suitable ratios for off-site research purposes that a regulatory authority believes are most important to its financial climate.

\section{Review of Literature}

Several scholars have used the CAMEL Model to study the performance of the Banking industry in emerging economies. According to the CAMEL Model, 65 Indian Banks' performance was evaluated for 2003-2004 and suggested that better quality of service, innovative products, and better bargains were advantageous because of its serious competition prevailing (Prasuna, 2004). The CAMEL existing and ten new Indian Private Banks for the five years, i.e., 2003-07, was assessed (Gupta, 2008). The study on the applicability of CAMEL standards and their subsequent influence on the quality of SBI groups concluded that the yearly screening of CAMEL enables the Commercial Bank to assess its financial condition and warns the authorities to take preventive measures it is sustainable (Misra and Aspal, 2012). The study analyses Commercial Banks' productivity in India for five years 2009-13 using the CAMEL methodology, suggesting that Commercial Banks' actual performance with CAMEL ratios is distinct (Gupta, 2014). An analysis of the performance of Indian Banks using the CAMEL Model for ten years of 2006-2007 to 2015-16 suggested that the general shareholder fund must be raised and demand liability minimized to obtain a good financial position (Subbiramani et al. 2018). A Study, attempt to assess and analyse the financial health and viability of CAMEL Methodology during 2011-2016 and noted that Liquidity of HDFC Bank, Indusland Bank, and ICICI Bank is worthy (Gupta, 2014). The study used the CAMEL Model to assess the financial performance adequacy of the State Bank Group. It noted that SBI needs to strengthen its role on Asset Quality and Capital Adequacy, SBBJ must boost its operational effectiveness, and SBP should improve the quality of its earnings (Kumar and Sharma, 2014). A CAMEL study of five Banks, namely ICICI, SBI, AXIS, HDFC, and BOI, shows that Public sector Banks, such as BOI, have performed exceptionally well on every CAMEL variable. While in the case of Private Sector Banks, underperformed by ICICI (Tatuskar, 2010). Based on the CAMEL ranking, for the ten-year data (2004-05 to 2013-14), Bank of Baroda and PNB, Commercial Banks are considered to become the most reliable Banks. The IDBI, Indian Bank, Canara Bank, and SBI are considered the average Bank (Kaur and Kaur, 2016).

We have previously fundamentally examined the performance of Public and Private sector Banks and shown that during 2011-2018, Private (ICICI and AXIS) performed better than Public (SBI and PNB) sector Banks (Jha, 2018, Jha, 2019b, Jha, 2019a, Jha and M, 2019). However, a comparative study on the financial results of these Banks lacks the CAMEL Model during 2011-2018. 


\section{Objective and Hypotheses of the Study}

\subsection{Objective}

The objective of the study is:

- To study a comparative financial analysis of two Public (SBI and PNB) and Private (ICICI and AXIS) Banks with CAMEL Model.

\subsection{Hypotheses}

Based on the objective, the hypotheses are formulated as:

$\mathrm{H}_{0}$ : There is no significant difference in financial performance of Public and Private sector Banks with CAMEL Model.

$\mathrm{H}_{1}$ : There is a significant difference in the financial performance of Public and Private sector Banks with CAMEL Model.

\section{Research Methodology}

\subsection{Data Collection}

The secondary data limited to 7 years (2011-2018) has been collected from annual reports, available on website of the respective banks.

\subsection{Tools and Techniques}

CAMEL Model has been used to evaluate these data. The following ratio has been taken under parameters of CAMEL Model.

Box 1: Mapping of CAMEL Parameters

\begin{tabular}{|c|c|c|c|c|}
\hline Capital Adequacy & Assets Quality & Management Capability & Earning Efficiency & Liquidity Status \\
\hline $\begin{array}{l}\text { Capital Adequacy } \\
\text { ratio }\end{array}$ & $\begin{array}{l}\text { Gross NPA / } \\
\text { Total Assets } \\
\text { ratio }\end{array}$ & $\begin{array}{l}\text { Business per } \\
\text { Employee }\end{array}$ & $\begin{array}{l}\text { Dividend Pay-out } \\
\text { ratio }\end{array}$ & $\begin{array}{l}\text { Liquid Assets/ Total } \\
\text { Assets ratio }\end{array}$ \\
\hline $\begin{array}{l}\text { Debt - Equity } \\
\text { ratio }\end{array}$ & $\begin{array}{l}\text { Net NPA / Total } \\
\text { Assets ratio }\end{array}$ & $\begin{array}{l}\text { Profit per } \\
\text { Employee }\end{array}$ & $\begin{array}{l}\text { Net Interest } \\
\text { Margin }\end{array}$ & $\begin{array}{l}\text { Liquid Assets / Total } \\
\text { Deposit ratio }\end{array}$ \\
\hline $\begin{array}{l}\text { Total Advances to } \\
\text { Total Assets ratio }\end{array}$ & $\begin{array}{l}\text { Gross NPA / } \\
\text { Total Advances } \\
\text { ratio }\end{array}$ & $\begin{array}{l}\text { Total Expenditure } \\
\text { to Total Income }\end{array}$ & $\begin{array}{l}\text { Net Profit } \\
\text { Margin }\end{array}$ & $\begin{array}{l}\text { Cash to Deposit } \\
\text { ratio. }\end{array}$ \\
\hline $\begin{array}{l}\text { Government } \\
\text { Securities to } \\
\text { Total Investment }\end{array}$ & $\begin{array}{l}\text { Net NPA / Total } \\
\text { Advances ratio }\end{array}$ & $\begin{array}{l}\text { Diversification } \\
\text { ratio }\end{array}$ & $\begin{array}{l}\text { Net profit to } \\
\text { Total Assets } \\
\text { ratio }\end{array}$ & Current ratio \\
\hline
\end{tabular}

contd. box 1 
Priyanka Jha and Subburaj Natarajan

\begin{tabular}{|c|c|c|c|c|}
\hline Capital Adequacy & Assets Quality & Management Capability & Earning Efficiency & Liquidity Status \\
\hline $\begin{array}{l}\text { Shareholder's } \\
\text { Fund/ Total } \\
\text { Advances }\end{array}$ & $\begin{array}{l}\text { Total Investment } \\
\text { to Total Assets } \\
\text { ratio }\end{array}$ & $\begin{array}{l}\text { Total Assets } \\
\text { Turnover ratio }\end{array}$ & $\begin{array}{l}\text { Operating Profit/ } \\
\text { Total Assets ratio }\end{array}$ & $\begin{array}{l}\text { Interest Expended to } \\
\text { Interest Earned ratio }\end{array}$ \\
\hline $\begin{array}{l}\text { Shareholder's } \\
\text { Fund/Total Assets } \\
\text { Net Worth Ratio }\end{array}$ & $\begin{array}{l}\text { Credit Deposit } \\
\text { ratio }\end{array}$ & $\begin{array}{l}\text { Return on Equity } \\
\text { ratio } \\
\text { Return on Assets ratio }\end{array}$ & $\begin{array}{l}\text { Interest Income/ } \\
\text { Total Income ratio } \\
\text { Growth in Profit }\end{array}$ & $\begin{array}{l}\text { Total Investment to } \\
\text { Total Deposit ratio } \\
\text { Government Securities } \\
\text { to Total Assets ratio }\end{array}$ \\
\hline
\end{tabular}

Source: Authors' Compilation for Literatures

\section{Data Analysis and Interpretation}

The CAMEL rating is based on a scale with a ranking, lower rating signifying a Bank that is more financially solid and less at risk. A composite rating of 1 indicates that a Bank has a strong performance and follows risk management guidelines. A Bank with a rating of 2 is financially healthy but has moderate flaws. A rating of 3 indicates that a supervisory concern is needed. On a rating of 4 , a Bank with unsound practices is considered dangerous due to substantial financial concerns. Rating 5 indicates that a Bank is fundamentally unsound and has poor risk management methods.

\subsection{Capital Adequacy and its Sub-parameters}

Table 1 show that, ICICI got rank (1), followed by AXIS (2), SBI (3) and PNB (4) in the Capital Adequacy ratio, Debt-Equity ratio. However, PNB was also able to retain the mandatory Capital Adequacy ratio but was ranked (4). PNB Bank uses maximum debt and also has a low Capital Adequacy Ratio. PNB got rank (1) followed by SBI (2), AXIS (3) and ICICI (4) in risk-free Government Securities. PNB has invested much of its overall investment in risk-free Government Securities, suggesting that it has an excellent risk-free investment policy.

Table 1: Average of Sub-parameters of Capital Adequacy

\begin{tabular}{lcccc}
\hline Year & SBI & PNB & ICICI & AXIS \\
\hline & & Capital Adequacy ratio & & \\
$2011-2012$ & 13.86 & 12.63 & 18.5 & 13.66 \\
$2012-2013$ & 12.92 & 12.72 & 18.74 & 17.00 \\
$2013-2014$ & 12.44 & 11.52 & 17.70 & 16.07 \\
$2014-2015$ & 12.00 & 12.21 & 17.00 & 15.09 \\
$2015-2016$ & 13.12 & 11.28 & 16.60 & 15.29 \\
$2016-2017$ & 13.11 & 11.66 & 17.40 & 14.95 \\
$2017-2018$ & 12.60 & 9.20 & 18.40 & 16.57 \\
Mean & 12.86 & 11.60 & 17.76 & 15.51 \\
Rank & 3 & 4 & 1 & 2 \\
\hline & & & & contd. table 1
\end{tabular}


Asset Quality Evaluation of Select Indian Banks: A Camel Model

\begin{tabular}{|c|c|c|c|c|}
\hline \multicolumn{5}{|c|}{ Debt-Equity ratio } \\
\hline 2011-2012 & 12.43 & 13.64 & 4.22 & 9.65 \\
\hline 2012-2013 & 12.16 & 11.98 & 4.38 & 7.63 \\
\hline 2013-2014 & 11.78 & 12.57 & 4.54 & 7.35 \\
\hline 2014-2015 & 12.27 & 12.83 & 4.50 & 7.22 \\
\hline 2015-2016 & 12.27 & 14.44 & 4.70 & 6.73 \\
\hline 2016-2017 & 10.85 & 14.86 & 4.90 & 7.43 \\
\hline 2017-2018 & 12.58 & 15.64 & 5.33 & 7.15 \\
\hline Mean & 12.04 & 13.70 & 4.65 & 7.59 \\
\hline Rank & 3 & 4 & 1 & 2 \\
\hline \multicolumn{5}{|c|}{ Total Advances/ Total Assets ratio } \\
\hline 2011-2012 & 64.96 & 64.12 & 53.57 & 59.43 \\
\hline $2012-2013$ & 66.76 & 64.47 & 54.07 & 57.84 \\
\hline 2013-2014 & 67.5 & 63.46 & 56.96 & 60.03 \\
\hline 2014-2015 & 63.48 & 63.07 & 59.98 & 60.85 \\
\hline 2015-2016 & 64.79 & 61.78 & 60.4 & 64.47 \\
\hline 2016-2017 & 58.06 & 58.24 & 60.15 & 62.03 \\
\hline 2017-2018 & 56.01 & 56.64 & 35.53 & 63.59 \\
\hline Mean & 63.08 & 61.68 & 54.38 & 61.17 \\
\hline Rank & 1 & 2 & 4 & 3 \\
\hline \multicolumn{5}{|c|}{ Government Securities/ Total Investment } \\
\hline 2011-2012 & 82.54 & 81.35 & 54.77 & 62.8 \\
\hline $2012-2013$ & 77.54 & 82.83 & 54.28 & 63.76 \\
\hline 2013-2014 & 78.25 & 78.10 & 54.17 & 61.32 \\
\hline 2014-2015 & 77.45 & 81.94 & 57.56 & 62.28 \\
\hline 2015-2016 & 77.63 & 80.14 & 70.33 & 71.43 \\
\hline 2016-2017 & 76.25 & 79.09 & 69.66 & 72.39 \\
\hline 2017-2018 & 80.95 & 76.86 & 69.72 & 67.77 \\
\hline Mean & 78.65 & 80.04 & 61.49 & 65.96 \\
\hline Rank & 2 & 1 & 4 & 3 \\
\hline \multicolumn{5}{|c|}{ Share-bolder's Fund/Total Advances } \\
\hline 2011-2012 & 9.68 & 9.47 & 23.81 & 13.44 \\
\hline 2012-2013 & 9.46 & 10.58 & 22.98 & 16.81 \\
\hline
\end{tabular}

Orissa Journal of Commerce, 42(1) (C) 2021 
Priyanka Jha and Subburaj Natarajan

\begin{tabular}{|c|c|c|c|c|}
\hline 2013-2014 & 9.78 & 10.28 & 21.62 & 16.61 \\
\hline 2014-2015 & 9.88 & 10.27 & 20.75 & 15.89 \\
\hline 2015-2016 & 9.86 & 9.29 & 20.62 & 15.69 \\
\hline 2016-2017 & 11.98 & 9.98 & 21.53 & 14.95 \\
\hline $2017-2018$ & 11.33 & 9.47 & 33.66 & 14.43 \\
\hline Mean & 10.28 & 9.90 & 23.57 & 15.40 \\
\hline Rank & 3 & 4 & 1 & 2 \\
\hline \multicolumn{5}{|c|}{ Share-holder's Fund/Total Assets } \\
\hline 2011-2012 & 6.29 & 6.07 & 12.75 & 7.99 \\
\hline 2012-2013 & 6.31 & 6.82 & 12.43 & 9.72 \\
\hline 2013-2014 & 6.6 & 6.52 & 12.31 & 9.97 \\
\hline 2014-2015 & 6.27 & 6.48 & 12.45 & 9.67 \\
\hline 2015-2016 & 6.39 & 5.74 & 12.45 & 10.12 \\
\hline 2016-2017 & 6.96 & 5.81 & 12.95 & 9.27 \\
\hline $2017-2018$ & 6.34 & 5.36 & 11.96 & 9.18 \\
\hline Mean & 6.45 & 6.11 & 12.47 & 9.42 \\
\hline Rank & 3 & 4 & 1 & 2 \\
\hline \multicolumn{5}{|c|}{ Return on Net Worth Ratio } \\
\hline 2011-2012 & 13.94 & 18.60 & 10.70 & 18.60 \\
\hline 2012-2013 & 14.26 & 15.64 & 12.48 & 15.64 \\
\hline 2013-2014 & 9.20 & 16.26 & 13.40 & 16.27 \\
\hline 2014-2015 & 10.20 & 16.46 & 13.90 & 16.47 \\
\hline 2015-2016 & 06.89 & 15.46 & 10.84 & 15.47 \\
\hline 2016-2017 & 05.56 & 06.59 & 9.81 & 06.6 \\
\hline $2017-2018$ & $(-2.98)$ & 0.43 & 6.44 & 0.44 \\
\hline Mean & 8.15 & 12.77 & 11.08 & 12.78 \\
\hline Rank & 4 & 2 & 3 & 1 \\
\hline
\end{tabular}

Source: Authors' Compilation

\subsection{Assets Quality and its Sub-parameters}

It has been observed in Sub-parameters of Assets Quality, Gross NPA /Total Assets ratio, Net NPAs to Total Assets ratio, Net NPA / Total Advances ratio AXIS is ranked (1) followed by ICICI (2), SBI (3) and PNB (4). It can be ensure that AXIS has the highest composite Asset Quality followed by ICICI, SBI and PNB. Efficiency of PNB is poor, it has maximum NPA. However, in case of Credit Deposit ratio, ICICI has performed better than AXIS, whereas SBI and PNB perform poor.

64

Orissa Journal of Commerce, 42(1) (C) 2021 
Asset Quality Evaluation of Select Indian Banks: A Camel Model

Table 2: Average of Sub-parameters of Assets Quality

\begin{tabular}{|c|c|c|c|c|}
\hline Year & $S B I$ & $P N B$ & ICICI & AXIS \\
\hline \multicolumn{5}{|c|}{ Gross NPA / Total Assets ratio (\%) } \\
\hline 2011-2012 & 2.97 & 1.90 & 2.00 & 0.63 \\
\hline 2012-2013 & 3.27 & 2.81 & 1.79 & 0.70 \\
\hline 2013-2014 & 3.44 & 3.43 & 1.77 & 0.82 \\
\hline 2014-2015 & 2.77 & 4.26 & 2.34 & 0.89 \\
\hline 2015-2016 & 4.35 & 8.36 & 3.64 & 1.16 \\
\hline 2016-2017 & 4.15 & 7.69 & 5.46 & 3.54 \\
\hline $2017-2018$ & 6.47 & 11.31 & 6.06 & 4.95 \\
\hline Mean & 3.91 & 5.68 & 3.29 & 1.81 \\
\hline Rank & 3 & 4 & 2 & 1 \\
\hline \multicolumn{5}{|c|}{ Net NPA / Total Assets ratio (\%) } \\
\hline 2011-2012 & 1.18 & 0.97 & 0.39 & 0.17 \\
\hline 2012-2013 & 1.40 & 1.51 & 0.42 & 0.21 \\
\hline 2013-2014 & 1.74 & 1.80 & 0.55 & 0.27 \\
\hline 2014-2015 & 1.35 & 2.56 & 0.97 & 0.29 \\
\hline 2015-2016 & 2.47 & 5.31 & 1.80 & 0.48 \\
\hline 2016-2017 & 2.15 & 4.54 & 3.27 & 1.43 \\
\hline 2017-2018 & 3.21 & 6.36 & 3.16 & 2.40 \\
\hline Mean & 1.92 & 3.29 & 1.50 & 0.75 \\
\hline Rank & 3 & 4 & 2 & 1 \\
\hline \multicolumn{5}{|c|}{ Gross NPA / Total Advances ratio (\%) } \\
\hline 2011-2012 & 4.45 & 2.93 & 3.62 & 1.06 \\
\hline 2012-2013 & 4.76 & 4.27 & 3.23 & 1.21 \\
\hline 2013-2014 & 4.97 & 5.24 & 3.03 & 1.367 \\
\hline 2014-2015 & 4.27 & 6.58 & 3.81 & 1.45 \\
\hline 2015-2016 & 6.52 & 12.91 & 5.85 & 1.78 \\
\hline 2016-2017 & 6.91 & 12.54 & 8.76 & 5.52 \\
\hline 2017-2018 & 10.91 & 18.42 & 9.90 & 7.50 \\
\hline Mean & 6.11 & 8.98 & 5.45 & 2.84 \\
\hline Rank & 3 & 4 & 2 & 1 \\
\hline \multicolumn{5}{|c|}{ Net NPA / Total Advances ratio (\%) } \\
\hline 2011-2012 & 1.82 & 1.53 & 0.73 & 0.28 \\
\hline 2012-2013 & 2.10 & 2.34 & 0.77 & 0.36 \\
\hline
\end{tabular}

contd. table 2 
Priyanka Jha and Subburaj Natarajan

\begin{tabular}{|c|c|c|c|c|}
\hline Year & $S B I$ & $P N B$ & ICICI & AXIS \\
\hline 2013-2014 & 2.57 & 2.84 & 0.97 & 0.45 \\
\hline 2014-2015 & 2.12 & 4.05 & 1.61 & 0.47 \\
\hline 2015-2016 & 3.81 & 8.60 & 2.98 & 0.75 \\
\hline 2016-2017 & 3.71 & 7.80 & 5.43 & 2.31 \\
\hline 2017-2018 & 5.73 & 11.22 & 5.43 & 3.78 \\
\hline Mean & 3.12 & 5.48 & 2.56 & 1.20 \\
\hline Rank & 3 & 4 & 2 & 1 \\
\hline \multicolumn{5}{|c|}{ Total Investment to Total Assets ratio (\%) } \\
\hline 2011-2012 & 23.38 & 26.76 & 33.69 & 32.63 \\
\hline 2012-2013 & 22.41 & 27.13 & 31.93 & 33.4 \\
\hline 2013-2014 & 22.22 & 26.12 & 29.77 & 29.63 \\
\hline 2014-2015 & 24.17 & 25.07 & 28.88 & 28.65 \\
\hline 2015-2016 & 21.12 & 23.65 & 22.26 & 23.22 \\
\hline 2016-2017 & 28.31 & 25.92 & 20.93 & 21.41 \\
\hline $2017-2018$ & 30.71 & 26.16 & 23.09 & 22.25 \\
\hline Mean & 24.61 & 25.83 & 27.22 & 27.31 \\
\hline Rank & 1 & 2 & 3 & 4 \\
\hline \multicolumn{5}{|c|}{ Credit Deposit ratio } \\
\hline 2011-2012 & 83.12 & 77.39 & 99.30 & 77.13 \\
\hline 2012-2013 & 86.93 & 78.84 & 99.19 & 77.97 \\
\hline 2013-2014 & 86.76 & 77.38 & 102.04 & 81.89 \\
\hline 2014-2015 & 82.44 & 75.90 & 107.17 & 87.17 \\
\hline 2015-2016 & 84.57 & 74.55 & 103.28 & 94.64 \\
\hline 2016-2017 & 76.83 & 67.47 & 94.73 & 90.03 \\
\hline 2017-2018 & 71.49 & 67.53 & 91.34 & 96.92 \\
\hline Mean & 81.73 & 74.15 & 99.57 & 86.53 \\
\hline Rank & 3 & 4 & 1 & 2 \\
\hline
\end{tabular}

Source: Authors' Compilation

\subsection{Management Capability and its Sub-parameters}

Table 3 showed that the highest contribution is made by SBI and got rank (1) followed by ICICI (2), AXIS (3) and PNB (4) in case of Profit per Employee. ICICI is ranked (1) followed by AXIS (2), SBI (3) and PNB (4) in Diversification ratio and Return on Assets ratio suggesting that the Bank earns more revenue than daily Banking operations from fee-based activities. AXIS is ranked (1), followed by ICICI (2), SBI (3) and PNB (4) in Return on Equity ratio, Earnings per Staff and Total Asset Turnover ratio (Total Income/Total Assets). 
Asset Quality Evaluation of Select Indian Banks: A Camel Model

Table 3: The Average of Management Capability and its Sub-parameters

\begin{tabular}{|c|c|c|c|c|}
\hline Year & $S B I$ & $P N B$ & $I C I C I$ & AXIS \\
\hline \multicolumn{5}{|c|}{ Business per Employee (in crore) } \\
\hline 2011-2012 & 07.98 & 11.32 & 7.08 & 12.76 \\
\hline 2012-2013 & 09.44 & 11.65 & 7.35 & 12.15 \\
\hline 2013-2014 & 10.64 & 12.83 & 7.47 & 12.3 \\
\hline 2014-2015 & 12.34 & 13.19 & 8.32 & 13.71 \\
\hline 2015-2016 & 14.11 & 13.59 & 9.43 & 14.84 \\
\hline 2016-2017 & 16.24 & 14.17 & 9.89 & 14.00 \\
\hline 2017-2018 & 16.70 & 14.74 & 10.78 & 14.84 \\
\hline Mean & 12.49 & 13.07 & 8.61 & 13.51 \\
\hline Rank & 3 & 2 & 4 & 1 \\
\hline \multicolumn{5}{|c|}{ Profit per Employee (in crore) } \\
\hline 2011-2012 & 0.53 & 0.08 & 0.11 & 0.14 \\
\hline 2012-2013 & 0.65 & 0.08 & 0.14 & 0.15 \\
\hline 2013-2014 & 0.49 & 0.05 & 0.14 & 0.15 \\
\hline 2014-2015 & 0.60 & 0.05 & 0.16 & 0.17 \\
\hline 2015-2016 & 0.47 & -0.06 & 0.14 & 0.18 \\
\hline 2016-2017 & 0.51 & 0.02 & 0.12 & 0.07 \\
\hline 2017-2018 & -0.24 & -0.17 & 0.08 & 0.00 \\
\hline Mean & 0.43 & 0.007 & 0.13 & 0.12 \\
\hline Rank & 1 & 4 & 2 & 3 \\
\hline \multicolumn{5}{|c|}{ Total Expenditure to Total Income (\%) } \\
\hline 2011-2012 & 90.31 & 87.99 & 84.25 & 84.53 \\
\hline 2012-2013 & 89.61 & 89.70 & 83.8 & 84.65 \\
\hline 2013-2014 & 92.97 & 93.01 & 82.03 & 83.66 \\
\hline 2014-2015 & 92.51 & 94.13 & 81.76 & 83.22 \\
\hline 2015-2016 & 94.81 & 109.08 & 85.71 & 83.67 \\
\hline 2016-2017 & 95.03 & 97.64 & 86.69 & 93.46 \\
\hline 2017-2018 & 102.47 & 121.6 & 90.64 & 99.51 \\
\hline Mean & 93.95 & 99.02 & 84.98 & 87.52 \\
\hline Rank & 3 & 4 & 1 & 2 \\
\hline \multicolumn{5}{|c|}{ Diversification ratio (\%) } \\
\hline 2011-2012 & 11.87 & 10.33 & 18.28 & 19.77 \\
\hline 2012-2013 & 11.82 & 9.14 & 17.24 & 19.42 \\
\hline
\end{tabular}

Orissa Journal of Commerce, 42(1) (C) 2021 
Priyanka Jha and Subburaj Natarajan

\begin{tabular}{|c|c|c|c|c|}
\hline Year & $S B I$ & $P N B$ & $I C I C I$ & AXIS \\
\hline 2013-2014 & 11.98 & 9.57 & 19.1 & 19.46 \\
\hline 2014-2015 & 12.9 & 11.28 & 19.87 & 19.08 \\
\hline 2015-2016 & 14.68 & 11.23 & 22.51 & 18.61 \\
\hline 2016-2017 & 16.81 & 15.92 & 26.48 & 20.79 \\
\hline 2017-2018 & 16.83 & 15.61 & 24.07 & 19.33 \\
\hline Mean & 13.84 & 11.86 & 21.07 & 19.49 \\
\hline Rank & 3 & 4 & 1 & 2 \\
\hline \multicolumn{5}{|c|}{ Total Income/ Total Assets (Total Assets Turnover ratio) (\%) } \\
\hline 2011-2012 & 9.05 & 08.88 & 8.39 & 9.6 \\
\hline $2012-2013$ & 8.66 & 09.63 & 9.02 & 9.91 \\
\hline 2013-2014 & 8.64 & 08.68 & 9.18 & 9.93 \\
\hline 2014-2015 & 8.54 & 08.65 & 9.48 & 9.50 \\
\hline 2015-2016 & 8.49 & 08.00 & 9.44 & 9.59 \\
\hline 2016-2017 & 7.79 & 07.80 & 9.54 & 9.35 \\
\hline 2017-2018 & 7.67 & 07.42 & 8.23 & 8.21 \\
\hline Mean & 8.40 & 8.43 & 9.04 & 9.44 \\
\hline Rank & 4 & 3 & 2 & 1 \\
\hline \multicolumn{5}{|c|}{ Return on Equity ratio (\%) } \\
\hline 2011-2012 & 14.36 & 18.52 & 11.10 & 21.22 \\
\hline 2012-2013 & 15.94 & 15.19 & 12.90 & 20.51 \\
\hline 2013-2014 & 10.49 & 9.69 & 13.70 & 18.23 \\
\hline 2014-2015 & 11.17 & 8.12 & 14.30 & 18.57 \\
\hline 2015-2016 & 7.74 & $(-11.20)$ & 11.30 & 17.49 \\
\hline 2016-2017 & 7.25 & 3.52 & 10.30 & 7.22 \\
\hline 2017-2018 & $(-3.78)$ & $(-32.85)$ & 6.60 & 0.53 \\
\hline Mean & 9.02 & 1.57 & 11.45 & 14.82 \\
\hline Rank & 3 & 4 & 2 & 1 \\
\hline \multicolumn{5}{|c|}{ Return on Assets ratio (\%) } \\
\hline 2011-2012 & 0.88 & 1.19 & 1.5 & 1.68 \\
\hline $2012-2013$ & 0.97 & 1.00 & 1.66 & 1.70 \\
\hline 2013-2014 & 0.65 & 0.64 & 1.76 & 1.78 \\
\hline 2014-2015 & 0.68 & 0.53 & 1.86 & 1.83 \\
\hline 2015-2016 & 0.46 & $(-0.61)$ & 1.49 & 1.72 \\
\hline 2016-2017 & 0.41 & 0.19 & 1.35 & 0.65 \\
\hline $2017-2018$ & $(-0.19)$ & $(-1.6)$ & 0.87 & 0.04 \\
\hline Mean & 0.55 & 0.19 & 1.49 & 1.34 \\
\hline Rank & 3 & 4 & 1 & 2 \\
\hline
\end{tabular}

Source: Authors' Compilation 
Asset Quality Evaluation of Select Indian Banks: A Camel Model

\subsection{Earning Capability and its Sub-parameters}

Table 4 shows that ICICI got rank (1), followed by SBI (2), AXIS (3) and PNB (4) in the Dividend Payout ratio, Net Profit/Total Income ratio, Net Profit to Total Assets ratio and Growth in Profit. AXIS is ranked $\left(1^{\text {st }}\right)$, led by ICICI $\left(2^{\text {nd }}\right)$ and SBI $\left(3^{\text {rd }}\right)$ and PNB $\left(4^{\text {th }}\right)$ in Net Interest Margin.

Table 4: Average of Earning Capability and its Sub-parameters

\begin{tabular}{|c|c|c|c|c|}
\hline Year & $S B I$ & $P N B$ & $I C I C I$ & AXIS \\
\hline \multicolumn{5}{|c|}{ Dividend Payout ratio } \\
\hline 2011-2012 & 18.98 & 14.28 & 32.35 & 15.54 \\
\hline 2012-2013 & 19.75 & 19.35 & 30.46 & 15.04 \\
\hline 2013-2014 & 19.13 & 10.65 & 29.77 & 15.09 \\
\hline 2014-2015 & 19.94 & 19.52 & 28.47 & 14.75 \\
\hline 2015-2016 & 20.03 & 0.00 & 32.83 & 14.46 \\
\hline 2016-2017 & 19.35 & 0.00 & 16.33 & 32.47 \\
\hline $2017-2018$ & Nil & Nil & 14.34 & Nil \\
\hline Mean & 16.74 & 9.11 & 26.36 & 15.33 \\
\hline Rank & 2 & 4 & 1 & 3 \\
\hline \multicolumn{5}{|c|}{ Net Interest Margin } \\
\hline 2011-2012 & 3.24 & 2.92 & 2.20 & 2.80 \\
\hline $2012-2013$ & 2.83 & 3.10 & 2.58 & 2.84 \\
\hline 2013-2014 & 2.74 & 2.93 & 2.77 & 3.12 \\
\hline 2014-2015 & 2.69 & 2.74 & 2.95 & 3.08 \\
\hline 2015-2016 & 2.52 & 2.29 & 2.95 & 3.20 \\
\hline 2016-2017 & 2.28 & 2.08 & 2.82 & 3.01 \\
\hline $2017-2018$ & 2.17 & 1.95 & 2.62 & 2.70 \\
\hline Mean & 2.63 & 2.57 & 2.69 & 2.96 \\
\hline Rank & 3 & 4 & 2 & 1 \\
\hline \multicolumn{5}{|c|}{ Net Profit/Total Income ratio } \\
\hline 2011-2012 & 9.68 & 12.00 & 15.75 & 15.47 \\
\hline $2012-2013$ & 10.39 & 10.30 & 17.19 & 15.35 \\
\hline 2013-2014 & 7.03 & 07.00 & 17.97 & 16.34 \\
\hline 2014-2015 & 7.48 & 05.86 & 18.24 & 16.78 \\
\hline $2015-2016$ & 5.18 & $(-07.44)$ & 14.29 & 16.33 \\
\hline 2016-2017 & 4.96 & 02.35 & 13.31 & 6.54 \\
\hline $2017-2018$ & $(-2.46)$ & $(-21.59)$ & 9.36 & 0.49 \\
\hline Mean & 6.03 & 1.21 & 15.15 & 12.47 \\
\hline Rank & 3 & 4 & 1 & 2 \\
\hline
\end{tabular}

Orissa Journal of Commerce, 42(1) (C) 2021 
Priyanka Jha and Subburaj Natarajan

\begin{tabular}{|c|c|c|c|c|}
\hline \multicolumn{5}{|c|}{ Net profit to Total Assets ratio } \\
\hline $2011-2012$ & 0.88 & 1.07 & 1.36 & 1.49 \\
\hline $2012-2013$ & 0.9 & 0.99 & 1.55 & 1.52 \\
\hline $2013-2014$ & 0.61 & 0.61 & 1.65 & 1.62 \\
\hline $2014-2015$ & 0.64 & 0.51 & 1.73 & 1.59 \\
\hline $2015-2016$ & 0.44 & $(-0.60)$ & 1.35 & 1.57 \\
\hline $2016-2017$ & 0.39 & 0.18 & 1.27 & 0.61 \\
\hline $2017-2018$ & $(-0.19)$ & $(-1.60)$ & 0.77 & 0.04 \\
\hline Mean & 0.52 & 0.16 & 1.38 & 1.20 \\
\hline Rank & 3 & 4 & 1 & 2 \\
\hline \multicolumn{5}{|c|}{ Operating Profit / Total Assets ratio (\%) } \\
\hline $2011-2012$ & 2.36 & 2.32 & 2.19 & 2.60 \\
\hline $2012-2013$ & 1.98 & 2.28 & 2.46 & 2.73 \\
\hline 2013-2014 & 1.79 & 2.07 & 2.79 & 2.99 \\
\hline 2014-2015 & 1.9 & 1.98 & 3.05 & 2.90 \\
\hline $2015-2016$ & 1.91 & 1.83 & 3.31 & 3.06 \\
\hline $2016-2017$ & 1.88 & 2.02 & 3.43 & 2.92 \\
\hline $2017-2018$ & 1.72 & 1.34 & 2.81 & 2.26 \\
\hline Mean & 1.93 & 1.97 & 2.86 & 2.78 \\
\hline Rank & 4 & 3 & 1 & 2 \\
\hline \multicolumn{5}{|c|}{ Interest Income/Total Income ratio } \\
\hline 2011-2012 & 88.13 & 89.67 & 81.72 & 80.23 \\
\hline 2012-2013 & 88.18 & 90.86 & 82.76 & 80.58 \\
\hline $2013-2014$ & 88.02 & 90.43 & 80.90 & 80.54 \\
\hline 2014-2015 & 87.10 & 88.72 & 80.13 & 80.92 \\
\hline $2015-2016$ & 85.32 & 88.77 & 77.49 & 81.39 \\
\hline $2016-2017$ & 83.19 & 84.08 & 73.52 & 79.21 \\
\hline $2017-2018$ & 83.18 & 84.39 & 75.93 & 80.67 \\
\hline Mean & 86.16 & 88.13 & 78.92 & 80.50 \\
\hline Rank & 2 & 1 & 4 & 3 \\
\hline \multicolumn{5}{|c|}{ Growth in Profit } \\
\hline 2011-2012 & Nil & Nil & Nil & Nil \\
\hline $2012-2013$ & 20.48 & $(-02.80)$ & 28.77 & 22.09 \\
\hline 2013-2014 & $(-22.78)$ & $(-29.60)$ & 17.84 & 20.06 \\
\hline 2014-2015 & 20.30 & $(-08.40)$ & 13.91 & 18.33 \\
\hline 2015-2016 & $(-24.05)$ & $(-229.83)$ & $(-12.97)$ & 11.78 \\
\hline 2016-2017 & 05.39 & 133.31 & 0.77 & $(-55.27)$ \\
\hline $2017-2018$ & $(-162.44)$ & $(-1027.64)$ & $(-30.85)$ & $(-92.5)$ \\
\hline Mean & $(-23.30)$ & $(-164.02)$ & 2.50 & $(-10.79)$ \\
\hline Rank & 3 & 4 & 1 & 2 \\
\hline
\end{tabular}

Source: Authors' Compilation 
Asset Quality Evaluation of Select Indian Banks: A Camel Model

\subsection{Liquidity Capability and its Sub-parameters}

Table 5, shows, in the Sub-parameters of Liquidity Capability of all 4 Banks get different rank. PNB is ranked (1), followed by ICICI (2), SBI (3) and AXIS (4) in Liquid Assets/ Total Assets ratio. In Liquid Assets/ Total Deposit ratio (\%) ICICI is ranked (1), followed by PNB (2), AXIS (3) and SBI (4). In Current ratio, PNB is ranked (1), followed by AXIS (2), ICICI (3) and SBI perform weak and got rank (4). Government Securities to Total Assets ratio calculates the Bank's Total Assets that are invested in Public securities. The higher ratio signifies the Bank's sound liquidity. In Government Securities, PNB is ranked (1), followed by SBI (2), AXIS (3) and ICICI (4). PNB is invested more in it, which are the most liquid and secure assets but affect the PNB earning efficiency. Like other marketing investment instruments, Government Securities do not offer high returns. In Government Securities, ICICI has spent less.

Table 5: The Average of Liquidity Capability and its Sub-parameters

\begin{tabular}{|c|c|c|c|c|}
\hline Year & $S B I$ & $P N B$ & $I C I C I$ & AXIS \\
\hline \multicolumn{5}{|c|}{ Liquid Assets/ Total Assets ratio (\%) } \\
\hline 2011-2012 & 7.28 & 6.29 & 7.65 & 4.88 \\
\hline $2012-2013$ & 7.33 & 5.67 & 7.72 & 6.00 \\
\hline 2013-2014 & 7.40 & 8.22 & 6.98 & 7.37 \\
\hline 2014-2015 & 8.54 & 9.27 & 6.55 & 7.81 \\
\hline 2015-2016 & 7.41 & 11.03 & 8.31 & 6.34 \\
\hline 2016-2017 & 6.36 & 12.26 & 9.81 & 8.36 \\
\hline $2017-2018$ & 5.55 & 12.47 & 9.57 & 6.29 \\
\hline Mean & 7.12 & 9.30 & 8.08 & 6.72 \\
\hline Rank & 3 & 1 & 2 & 4 \\
\hline \multicolumn{5}{|c|}{ Liquid Assets/ Total Deposit ratio (\%) } \\
\hline 2011-2012 & 9.31 & 7.59 & 14.18 & 6.33 \\
\hline $2012-2013$ & 9.55 & 6.93 & 14.15 & 8.09 \\
\hline 2013-2014 & 9.51 & 10.02 & 12.51 & 10.05 \\
\hline 2014-2015 & 11.09 & 11.16 & 11.70 & 11.20 \\
\hline $2015-2016$ & 9.68 & 13.31 & 14.21 & 9.31 \\
\hline $2016-2017$ & 8.41 & 14.2 & 15.45 & 12.12 \\
\hline $2017-2018$ & 7.09 & 14.86 & 15.00 & 9.58 \\
\hline Mean & 9.23 & 11.15 & 13.88 & 9.52 \\
\hline Rank & 4 & 2 & 1 & 3 \\
\hline \multicolumn{5}{|c|}{ Cash to Deposit ratio } \\
\hline 2011-2012 & 5.18 & 4.87 & 8.00 & 4.86 \\
\hline $2012-2013$ & 5.47 & 4.57 & 6.51 & 5.86 \\
\hline
\end{tabular}

Orissa Journal of Commerce, 42(1) (C) 2021 
Priyanka Jha and Subburaj Natarajan

\begin{tabular}{|c|c|c|c|c|}
\hline Year & SBI & $P N B$ & ICICI & AXIS \\
\hline 2013-2014 & 6.09 & 4.93 & 6.57 & 6.07 \\
\hline 2014-2015 & 7.34 & 4.83 & 7.10 & 6.15 \\
\hline 2015-2016 & 7.49 & 4.79 & 6.43 & 6.25 \\
\hline 2016-2017 & 6.26 & 4.05 & 6.47 & 7.45 \\
\hline 2017-2018 & 5.56 & 4.48 & 5.90 & 7.82 \\
\hline Mean & 6.19 & 4.64 & 6.71 & 6.35 \\
\hline Rank & 3 & 4 & 1 & 2 \\
\hline \multicolumn{5}{|c|}{ Current ratio } \\
\hline 2011-2012 & 12.58 & 24.58 & 17.61 & 22.00 \\
\hline 2012-2013 & 12.66 & 23.01 & 11.23 & 20.62 \\
\hline 2013-2014 & 14.37 & 26.72 & 11.88 & 19.38 \\
\hline 2014-2015 & 11.21 & 26.07 & 14.34 & 21.72 \\
\hline 2015-2016 & 11.08 & 30.99 & 15.92 & 26.47 \\
\hline 2016-2017 & 12.22 & 32.93 & 17.59 & 17.83 \\
\hline 2017-2018 & 14.08 & 25.79 & 22.13 & 20.33 \\
\hline Mean & 12.60 & 27.15 & 15.81 & 21.19 \\
\hline Rank & 4 & 1 & 3 & 2 \\
\hline \multicolumn{5}{|c|}{ Interest Expended to Interest Earned ratio (\%) } \\
\hline 2011-2012 & 59.35 & 63.22 & 68.00 & 63.55 \\
\hline 2012-2013 & 62.95 & 64.54 & 65.40 & 64.44 \\
\hline 2013-2014 & 63.85 & 62.64 & 62.70 & 61.00 \\
\hline 2014-2015 & 63.38 & 64.25 & 61.21 & 59.91 \\
\hline 2015-2016 & 65.24 & 67.71 & 56.76 & 58.93 \\
\hline 2016-2017 & 64.75 & 68.29 & 59.86 & 59.38 \\
\hline 2017-2018 & 66.05 & 68.90 & 58.11 & 59.33 \\
\hline Mean & 63.65 & 65.65 & 61.72 & 60.93 \\
\hline Rank & 3 & 4 & 2 & 1 \\
\hline \multicolumn{5}{|c|}{ Total Investment to Total Deposit ratio } \\
\hline 2011-2012 & 29.91 & 32.31 & 62.45 & 42.34 \\
\hline 2012-2013 & 29.18 & 33.17 & 58.57 & 45.02 \\
\hline 2013-2014 & 28.56 & 31.85 & 53.33 & 40.42 \\
\hline 2014-2015 & 31.39 & 30.17 & 51.6 & 41.04 \\
\hline 2015-2016 & 27.57 & 28.54 & 38.06 & 34.08 \\
\hline 2016-2017 & 27.46 & 30.03 & 32.96 & 31.08 \\
\hline 2017-2018 & 39.20 & 31.19 & 36.19 & 33.92 \\
\hline Mean & 30.46 & 31.03 & 47.59 & 38.27 \\
\hline Rank & 4 & 3 & 1 & 2 \\
\hline
\end{tabular}

72

Orissa Journal of Commerce, 42(1) (C) 2021 
Asset Quality Evaluation of Select Indian Banks: A Camel Model

\begin{tabular}{lcccc}
\hline \multicolumn{5}{c}{ Government Securities to Total Assets ratio } \\
\hline $2011-2012$ & 19.30 & 21.77 & 18.45 & 20.49 \\
$2012-2013$ & 17.37 & 22.47 & 17.33 & 21.29 \\
$2013-2014$ & 17.39 & 20.40 & 16.13 & 18.17 \\
$2014-2015$ & 18.72 & 20.54 & 16.62 & 17.84 \\
$2015-2016$ & 16.40 & 18.95 & 15.65 & 16.59 \\
$2016-2017$ & 21.58 & 20.50 & 14.58 & 15.50 \\
$2017-2018$ & 24.86 & 20.10 & 16.10 & 15.08 \\
Mean & 19.37 & 20.67 & 16.40 & 17.85 \\
Rank & 2 & 1 & 4 & 3 \\
\hline
\end{tabular}

Source: Authors' Compilation

\subsection{Composite Ranking of CAMEL's Parameters}

Based on the study (Table 6), ICICI and AXIS earned the same rank however SBI and PNB got lower rank (3) and (4) respectively in Composite Capital Adequacy (1.5). It can be ensure that AXIS (1) has the highest Composite Asset Quality and Management Efficiency followed by ICICI (2), SBI (3) and PNB (4). Efficiency of PNB is poorer, it has maximum NPA. However, in case of Credit Deposit ratio, ICICI has performed better than AXIS, whereas SBI and PNB perform poor. ICICI and AXIS is also a strong performer in Composite Earning Capability than SBI and PNB. PNB is ranked (1), followed by ICICI (2) and AXIS (3). SBI (4) in Composite Liquidity Capability.

Table 6: The Composite Ranking of CAMEL's Parameters

\begin{tabular}{|c|c|c|c|c|}
\hline Parameters & $S B I$ & $P N B$ & ICICI & AXIS \\
\hline \multicolumn{5}{|c|}{ Capital Adequacy } \\
\hline Capital Adequacy ratio & 3 & 4 & 1 & 2 \\
\hline Debt - Equity ratio & 3 & 4 & 1 & 2 \\
\hline Total Advances to Total Assets ratio & 1 & 2 & 4 & 3 \\
\hline Government Securities to Total Investment & 2 & 1 & 4 & 3 \\
\hline Share-Holder's Fund/ Total advances & 3 & 4 & 1 & 2 \\
\hline Share-holder's Fund/Total Assets & 3 & 4 & 1 & 2 \\
\hline Net Worth Ratio & 4 & 2 & 3 & 1 \\
\hline Composite & 2.71 & 3 & 2.14 & 2.14 \\
\hline Rank & 3 & 4 & 1.5 & 1.5 \\
\hline \multicolumn{5}{|c|}{ Assets Quality } \\
\hline Gross NPA /Total Assets ratio (\%) & 3 & 4 & 2 & 1 \\
\hline Net NPA /Total Assets ratio ( $\%)$ & 3 & 4 & 2 & 1 \\
\hline Gross NPA / Total Advances ratio (\%) & 3 & 4 & 2 & 1 \\
\hline
\end{tabular}

Orissa Journal of Commerce, 42(1) C 2021 
Priyanka Jha and Subburaj Natarajan

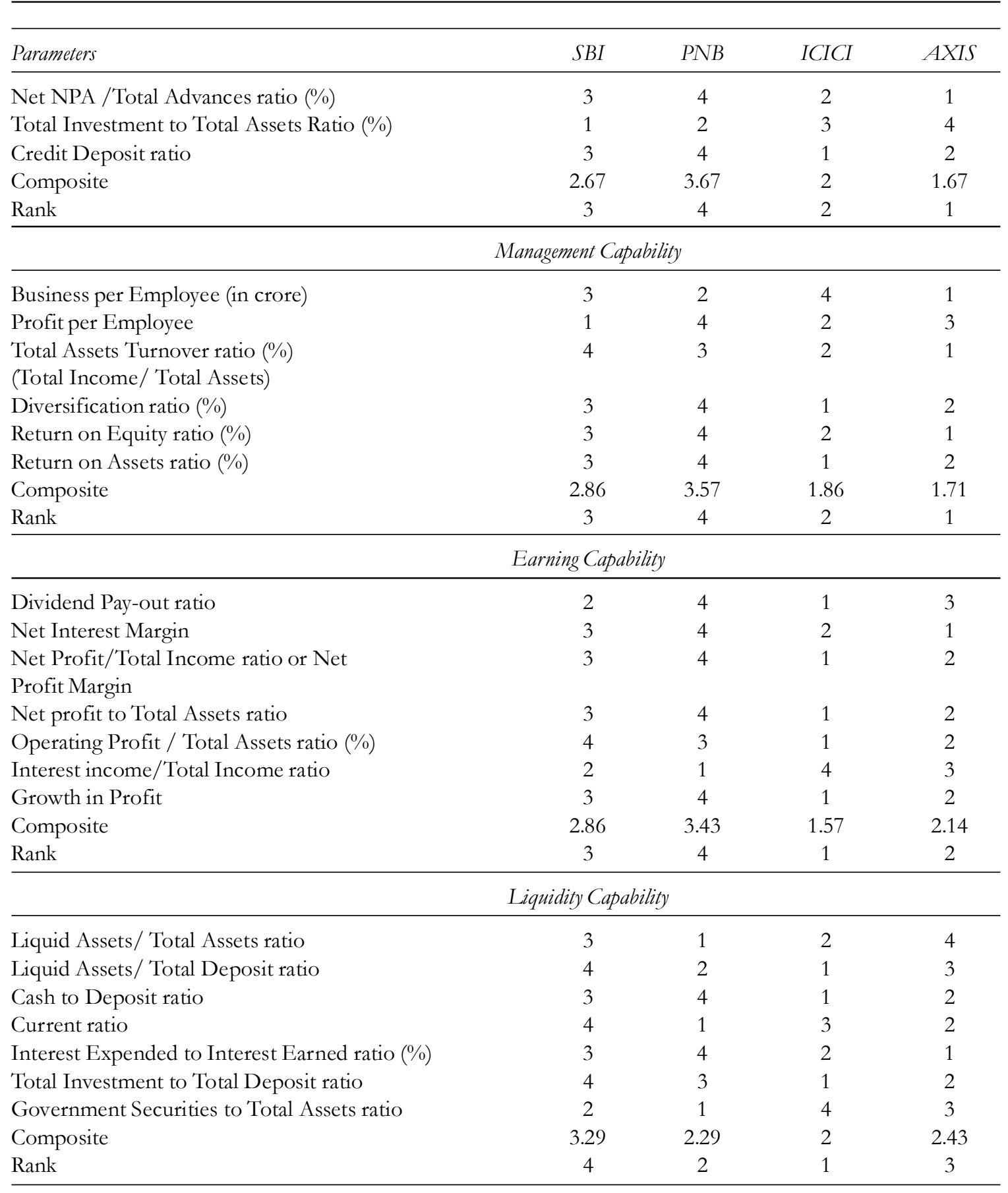

Source: Authors' Compilation 
Asset Quality Evaluation of Select Indian Banks: A Camel Model

\subsection{Overall Performance of Banks}

Based on the study (Table 7), the overall performance of the ICICI is excellent, and ranked (1) among four Banks followed by AXIS (2), SBI (3) and PNB (4). PNB performed poorly in the overall ranking. However, PNB was also able to retain the mandatory Capital Adequacy ratio but acquired lower ranked. The weak performance on Capital Adequacy ratio, Debt-Equity ratio, Shareholder's fund to Total Advances, Shareholder's Fund to Total Assets are reasons behind PNB's lowest rating. ICICI and AXIS perform better on the above financial parameters, suggesting that the Bank focuses on utilising revenue-generating tools to account for government standards. Nevertheless, SBI and PNB performed better in the remaining two parameters, i.e., Government Securities to Total Investment and Total advance to Total Assets ratio. It advocated that the Public Bank focuses primarily on the protection of the funds of depositors. PNB has invested more in Government Securities, which are the most liquid and secure assets but affect the PNB Earning Efficiency. Like other marketing investment instruments, Government Securities do not offer high returns. In Government Securities, ICICI has spent minimum.

Table 7: Composite Ranking (Overall Performance) of Banks

\begin{tabular}{lcccc}
\hline & $S B I$ & $P N B$ & $I C I C I$ & AXIS \\
\hline $\mathrm{C}$ & 2.71 & 3 & 2.14 & 2.14 \\
$\mathrm{~A}$ & 2.67 & 3.67 & 2 & 1.67 \\
$\mathrm{M}$ & 2.86 & 3.57 & 1.86 & 1.71 \\
$\mathrm{E}$ & 2.86 & 3.43 & 1.57 & 2.14 \\
$\mathrm{~L}$ & 3.29 & 2.29 & 2 & 2.43 \\
Mean & 2.88 & 3.19 & 1.91 & 2.02 \\
Rank & 3 & 4 & 1 & 2 \\
\hline
\end{tabular}

Source: Authors' Compilation

\section{Conclusion}

The CAMEL Model is an important technique for assessing a bank's financial soundness and recommending essential solutions for the remedy of a Bank's deficiencies. An attempt has been made to evaluate the financial soundness of select Indian Banks in this study. Two large Public (SBI and PNB) and Private (ICICI and AXIS) Sector Banks are included in the sample of selected Banks. The CAMEL Model was used to rank the financial institution, which included crucial factors such as Capital Adequacy, Asset Quality, Management Efficiency, Earnings Quality, and Liquidity.

The findings of this study showed that during 2011-2018, there is a significant difference in the performance of Public (SBI and PNB) and Private (ICICI and AXIS) sector Banks. As a result, the null hypothesis is rejected and the alternative hypothesis is accepted. Private Banks are better defenders than Public Banks, which have attained the least position in all parameter of the CAMEL Model. According to the CAMEL rating, ICICI overall performance is excellent, and gets a high CAMEL rating (ranked 1). In the CAMEL Model, performance of AXIS is also outstanding (ranked 2). On the 
contrary, the financial performance of Public Banks is not up to the mark, and must be continuously monitored to ensure their viability. Furthermore, the Banks with the lowest ranking must improve their performance in order to meet the acceptable requirements.

\section{Implications of the Study}

This study highlights the efficacy of four major Indian Banks in terms of competitiveness. These findings may be considerable use for Banking Institutions, policy makers and academic researchers. This study will assist decision-makers to pay more attention to the key Banking activities that will allow the Bank perform better and can increased their rank while compared to other Banks. From an academic point of view, in analysing the financial performance of leading Commercial Banks, this study offers a whole new viewpoint. It can be determined that the current study will assist the decision-makers of Indian Public and Private sector Banks and other groups of Indian Banking sector to concentrate on financial performance and thus increase its efficiency and profitability.

\section{Acknowledgments}

We would like to express our gratitude to the Department of Management, Sarvepalli Radhakrishnan University, Bhopal, India, for offering this research opportunity.

\section{References}

Altan, M., Yusufazari, H. and Bedük, A (2014). Performance analysis of Banks in Turkey using CAMEL Model, Proceedings of International Academic Conferences, 2014. International Institute of Social and Economic Sciences.

Getahun, M. (2015). Analyzing financial performance of Commercial Banks in Ethiopia: CAMEL Model”, Unpublished Master s Thesis. Addis Ababa University.

Gupta, R. (2008). "A CAMEL Model Analysis of Private Sector Banks in India”, Journal of Gyan Management, 2(1), 3-8.

Gupta, R. (2014). An analysis of Indian Public sector Banks using CAMEL Model, IOSR Journal of Business and Management, 16(1), 94-102.

Jha, P. (2018). Analyzing Financial Performance (2011-2018) of Public Sector Banks (PNB) and Private Sector Banks (ICICI) in India, ICTACT Journal on Management Studies, 4(3), 793-799.

Jha, P. (2019a). Analysis of Non-Performing Assets (NPAs) Among SBI Bank and ICICI Bank for Operating 2011-2018, Asian Journal of Research in Banking and Finance, 9(1), 1-13.

Jha, P. (2019b). A Comparative Statistical Approach towards Analysis of Non-performing assets (NPAs) among PNB and ICICI Bank operating 2011-2018. Research Journal of Management, 8(2), 16-27.

Jha, P. and Bhargav M. (2019). Assessing the Financial Status of SBI and ICICI Bank Operative 2011-2018, Apeejay-Journal of Management Sciences and Technology, 6(2), 8-19.

Jha P, Subburaj N. (2020). Fundamental and Technical Analysis of Indian Banks for operating 2011-2018. Shodh Sanchar Bulletein, 10(40):23-27.

Kaur, H. V. (2010). Analysis of Banks in India-A CAMEL Model. Global Business Review, 11(2), 257-280.

Kaur, J. and KAUR, H. V. (2016). CAMEL analysis of selected Public sector Banks. 13th National Conference on Management, Information Technology and Engineering (GJ-NatConMITE 2016). 
Asset Quality Evaluation of Select Indian Banks: A Camel Model

Kumar, S. \& SHARMA, R. (2014). Performance analysis of top Indian Banks through CAMEL Model, International Journal of Advanced Research in Management and Social Sciences, 3(7), 81-92.

Misra, D. S. and Aspal, P. (2012). A CAMEL Model analysis of state Bank group, Proceedings of 19th International Business Research Conference.

Prasuna, D. G. (2004). Performance Snapshot 2003-04. Chartered Financial Analyst, 10(11), 6-13.

Said, M. \& Saucier, P. (2003).Liquidity, solvency, and efficiency: An empirical analysis of the Japanese Banks’ distress, Journal of Oxford, 5(3), 354-358.

Sarker, A. (2005). CAMEL rating system in the context of Islamic Banking: A proposed 'S'for Shariah framework, Journal of Islamic Economics and Finance, 1(1), 78-84.

Subbiramani, M, Ranjith, G. and Balagurusamy, A. (2018). A CAMEL analysis on performance of Public and Private sector Banks (with special reference to Canara Bank and HDFC Bank), International Journal of Computational Research and Development, 3(1), 44-49

Tatuskar, S. (2010). A Study of Financial Performance of Select Indian Scheduled Commercial Banks Using CAMEL Methodology for 2006-2010, International Journal of Research in Commerce and Management, 1(1), 105121.

Tiwari, C. and Sontakke, R. (2013). Trend Analysis of Non Performing Asset in Scheduled Commercial Banks in India, International Journal of Application or Innovation in Engineering \& Management.

Triner, G. D. (2000). Banking and economic development: Brazil, 1889-1930, Palgrave New York.

Tripathi, D., Meghani, K. and Mahajan, S. (2014). Financial performance of AXIS Bank and Kotak Mahindra Bank in the post reform era: analysis on CAMEL Model, Ecoomics and Applied Management Research, 1(2), 2349-5677. 